\title{
Polynomial time algorithms to determine weakly reversible realizations of chemical reaction networks
}

\author{
János Rudan ${ }^{1}$, Gábor Szederkényi ${ }^{1,2}$, Katalin M. Hangos ${ }^{2,3}$, Tamás Péni ${ }^{2}$ \\ ${ }^{1}$ Faculty of Information Technology, Pázmány Péter Catholic University, \\ Práter u. 50/a, Budapest, H-1083, Hungary \\ ${ }^{2}$ Process Control Research Group, Systems and Control Laboratory, \\ Institute for Computer Science and Control (MTA SZTAKI), \\ Hungarian Academy of Sciences, P.O. Box 63., Budapest, H-1518, Hungary \\ ${ }^{3}$ Department of Electrical Engineering and Information Systems, \\ University of Pannonia, H-8200 Veszprém, Egyetem u. 10. \\ e-mail: rudan.janos@itk.ppke.hu, szederkenyi@itk.ppke.hu, \\ hangos@scl.sztaki.hu, pt@scl.sztaki.hu
}

\begin{abstract}
Weak reversibility is a crucial structural property of chemical reaction networks with mass action kinetics, because it has major implications related to the existence, uniqueness and stability of equilibrium points and to the boundedness of solutions. In this paper, we present two new algorithms to find dynamically equivalent weakly reversible realizations of a given chemical reaction network. They are based on linear programming (LP) and thus have polynomial time-complexity. Hence, these algorithms can deal with large-scale biochemical reaction networks, too. Furthermore, one of the methods is able to deal with linearly conjugate networks.
\end{abstract}

Keywords: chemical reaction networks, weak reversibility, dynamical equivalence, linear conjugacy, optimization

\section{Introduction}

The analysis of the structural properties and dynamical behaviour of biologically motivated kinetic systems is a quickly developing field. While determining the exact parameters in such a network can be difficult due to the complexity of the described system or imperfect data, it is known that there are several important properties that only depend on the structure of the model. Moreover, the reaction graph structure corresponding to a given kinetic dynamics is generally non-unique. These facts motivate us to construct algorithms that can compute 
kinetic systems with preferred structures (e.g. weakly reversible, minimal or maximal number of reactions, etc) that may provide useful information about the dynamical behaviour of the system.

By chemical reaction networks (CRNs), we mean deterministic kinetic systems obeying the mass action law. Being smooth nonlinear systems, all important qualitative phenomena in nonlinear dynamics (such as stable/unstable equilibria, limit cycles or even chaos) may appear in their dynamical behaviour $[1,2]$. This kinetic system form is widely used to describe nonnegative models in the fields of (bio)chemistry, population and epidemic dynamics and economy as well $[3,4]$.

The phenomena of macro-equivalence or dynamical equivalence [5] describes the fact that several reaction networks having different structure and/or parameters can produce the same dynamical behaviour. Computation of dynamically equivalent structures with certain properties is detailed in $[6,7,8,9]$ where a mixed integer linear programming (MILP)-based solution framework has been proposed. The solution of an MILP problem is generally NP-hard. Hence, solving such optimization problems can be computationally very expensive which seriously limits the size of the treatable networks. One may overcome this problem by tracing back the original MILP problem to simple linear programming (LP), if possible. This approach is followed in [10], where LP-based methods are presented to compute dynamically equivalent realizations containing minimal and maximal number of reactions.

Roughly speaking, weak reversibility means that all components of the reaction graph are strongly connected components. This property has a crucial role in the theory of CRNs, since it connects structural properties of the reaction graph to qualitative features of the dynamical behaviour of the reaction network which is especially useful in the deficiency zero and deficiency one cases. As it is formulated in the Deficiency Zero Theorem [11] for a CRN having zero deficiency and a weakly reversible structure, there exists precisely one asymptotically stable equilibrium point in each stoichiometric compatibility class. According to the Boundedness conjecture for which no counterexamples have been found, the solutions of any weakly reversible CRN are bounded. The conjecture was proved in [12] for the single linkage class case. Moreover, there exist important general results about the existence of equilibrium points in weakly reversible reaction networks $[13,14]$. Several MILP-based algorithms dealing with the computation of weakly reversible realizations of reaction networks were presented in $[15,16,17]$. However, these methods do not scale up very well with the size of the network due to the integer variables in the optimization.

Linear conjugacy can be considered as an extension of dynamical equivalence where a diagonal state transformation having strictly positive values is applied between the solutions of linearly conjugate CRNs [18]. This means that the trajectories of the networks can be related by a linear transformation. Two linearly conjugate networks can have different deficiency values, therefore, linear conjugacy gives us additional degrees of freedom to computationally find a 
reaction network having similar dynamical behaviour than the original studied one, but with a more advantageous (smaller) deficiency value. In $[15,16]$ MILP-based methods are presented to compute weakly reversible, linearly conjugate realizations. These methods are further developed in [17] to be able to find weakly reversible linearly conjugate CRN structures with minimal deficiency. Again, the computational complexity of the MILP problem often seriously restricts the problem size if reasonable time limits are concerned. Therefore, the aim of this paper is to present purely LP-based methods for computing dynamically equivalent and linearly conjugate weakly reversible $\mathrm{CRN}$ structures that can cope with large networks possibly containing several hundreds of complexes and reactions.

The structure of the paper is the following. In Section 2, the basic tools and notations are described for modelling CRNs. In Section 3 the existing computational methods for determining weakly reversible realizations are shortly reviewed and Section 4 contains the detailed description of the new methods. In Section 5, the computational results corresponding to the proposed methods are presented, while Section 6 contains the summary and conclusions of the work.

\section{Basic concepts and notions related to CRNs}

In this Section, the structural and dynamical description of CRNs are introduced based on $[6,7,8]$. Besides the notations, some important properties are also recalled related to the scope of the current work.

\subsection{Chemical Reaction Networks}

The set $\mathcal{S}=\left\{X_{i}, \ldots, X_{n}\right\}$ represents the $n$ chemical species contained in a given CRN. The concentrations of the species denoted by $x_{i}=\left[X_{i}\right], i=1, \ldots, n$ form the state vector $\mathbf{x} \in \mathbb{R}^{n}$ of the system. The whole system obeys the mass action law and, therefore, all the values of the states are nonnegative [3]. Chemical complexes are formally represented as nonnegative linear combinations of the species:

$$
C_{j}=\sum_{i=1}^{n} \alpha_{i, j} X_{i} \text { for } j=1, \ldots, m,
$$

where $m$ is the number of the complexes in the network, and $\alpha_{i, j}$ for $i=1, \ldots, n$, are the nonnegative integer stoichiometric coefficients of the $j$ th complex. An elementary reaction step, where the source complex $C_{j}=\sum_{i=1}^{n} \alpha_{i, j} X_{i}$ is transformed into the product complex $C_{l}=$ $\sum_{i=1}^{n} \beta_{i, l} X_{i}$ is denoted by

$$
C_{j} \rightarrow C_{l}
$$

The reaction rate corresponding to reaction (2) can be written according to the mass action law as:

$$
\rho_{j, l}(\mathbf{x})=k_{j, l} \prod_{i=1}^{n} x_{i}^{\alpha_{i, j}}
$$


where $k_{j, l}>0$ is the reaction rate coefficient.

If for any $i \neq l$ both reactions $C_{i} \rightarrow C_{l}$ and $C_{l} \rightarrow C_{i}$ are present in the network, they are handled as separate elementary reactions. It is also required from the above model class that $C_{i} \neq C_{j}$ for $i \neq j, i, j=1, \ldots, m$, and self-reactions (i.e. loop edges) of the form $C_{i} \rightarrow C_{i}$ are not allowed for $i=1, \ldots, m$.

\subsection{Graph representation}

A CRN can be represented as a weighted, directed graph $D=\left(V_{d}, E_{d}\right)$ consisting of a finite nonempty set $V_{d}$ of vertices and a finite set $E_{d}$ containing ordered pairs of distinct vertices called directed edges. The complexes are represented by the vertices, i.e. $V_{d}=\left\{C_{1}, \ldots C_{m}\right\}$, and the edges stand for the reactions: $\left(C_{j}, C_{l}\right) \in E_{d}$ if complex $C_{j}$ is transformed to $C_{l}$ in one of the reactions in the network. The weight of the edge $\left(C_{j}, C_{l}\right)$ is the reaction rate coefficient $k_{j, l}$. By the structure of a CRN we mean the unweighted directed graph of the reaction network.

\subsection{ODE-based description}

From the several different possibilities, we will use the following factorization of the right hand side of the kinetic ODEs describing the dynamics of the concentrations (see, e.g. $[19,6]$ ):

$$
\dot{\mathbf{x}}=Y \cdot A_{k} \cdot \psi(\mathbf{x})
$$

where $\mathbf{x} \in \mathbb{R}^{n}$ is the vector of specie concentrations. $Y \in \mathbb{R}^{n \times m}$ is the complex composition matrix in which the $j$ th column contains the stoichiometric coefficients of complex $C_{j}$, i.e. $Y_{i, j}=\alpha_{i, j}$. The vector mapping $\psi=\left[\begin{array}{lll}\psi_{1} & \ldots & \psi_{m}\end{array}\right]^{T} \in \mathbb{R}^{n} \rightarrow \mathbb{R}^{m}$ is defined as:

$$
\psi_{j}(\mathbf{x})=\prod_{i=1}^{n} x_{i}^{Y_{i, j}}, \quad j=1, \ldots, m
$$

Matrix $A_{k}$ describes the reaction graph as follows:

$$
\left[A_{k}\right]_{i, j}=\left\{\begin{array}{l}
k_{j, i}, \text { if } i \neq j \text { and reaction } C_{j} \rightarrow C_{i} \text { is present in the CRN } \\
0, \text { if } i \neq j \text { and } C_{j} \rightarrow C_{i} \text { is not present in the CRN }
\end{array}\right.
$$

Moreover, the non-positive diagonal elements of $A_{k}$ are given by

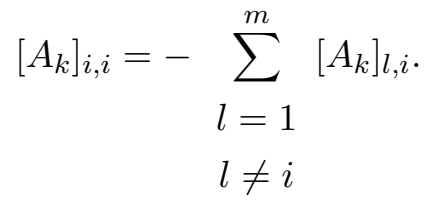

Hence, $A_{k}$ is a Metzler-type column conservation matrix (that is actually the negative transpose of the Laplacian matrix of the reaction graph), often called the Kirchhoff matrix of the CRN. 


\subsection{Dynamical equivalence and linear conjugacy of reaction networks}

A set of polynomial ODEs is called kinetic, if it can be written in the form (4), where $Y$ contains pairwise different columns of nonnegative integers, eq.(5) holds, and $A_{k}$ is a Kirchhoff matrix. Necessary and sufficient conditions of the kinetic property for polynomial systems with a constructive proof were first given in [20] (see also [21]). Let us introduce the matrix $M$ for the monomial coefficients of the model (4), i.e.

$$
M=Y \cdot A_{k} .
$$

Using the above notation, (4) can be written as

$$
\dot{\mathbf{x}}=M \cdot \psi(\mathbf{x}) .
$$

It is known that the factorization (8) is generally not unique (even if $Y$ is fixed), therefore the CRNs defined by the pairs $\left(Y^{(1)}, A_{k}^{(1)}\right)$ and $\left(Y^{(2)}, A_{k}^{(2)}\right)$ are called dynamically equivalent realizations of the kinetic system in eq. (9) (or that of each other) if $Y^{(i)}$ are valid complex composition matrices (a complex composition matrix is called valid if it contains nonnegative integer elements and there are no identical columns in it), $A_{k}^{(i)}$ are Kirchhoff for $i=1, \ldots, 2$, and

$$
Y^{(1)} \cdot A_{k}^{(1)}=Y^{(2)} \cdot A_{k}^{(2)}=M .
$$

It is known from the literature that the kinetic property of a system of ODEs is generally preserved up to the re-ordering and positive scaling of the state variables [22]. Therefore, in [18] the notion of linear conjugacy was introduced, where two CRNs are called linearly conjugate if (in the case of appropriate initial conditions) there is a positive linear diagonal mapping between the solutions of the corresponding kinetic ODEs. Linear conjugacy is the extension of dynamical equivalence and it is also clear that the qualitative properties of the solutions (number and stability of equilibirum points, persistence/extinction of species, dimensions of invariant spaces etc.) of two linearly conjugate CRNs are always the same.

\subsection{Weak reversibility}

The existence of a dynamically equivalent or linearly conjugate weakly reversible CRN realization can be useful in the analysis of the qualitative dynamical properties of the system [15]. From a graph-theoretic point of view, weak reversibility holds if and only if all components (i.e. linkage classes) of the reaction graph are strongly connected components (i.e. if there exists a directed path between nodes $C_{i}$ and $C_{j}$ then there exists a directed path from $C_{j}$ to $C_{i}$ ). Additionally, it is known that a CRN with a Kirchhoff matrix $A_{k}$ is weakly reversible if and only if there exists a strictly positive vector in the kernel of $A_{k}$ [15], i.e.

$$
\begin{aligned}
A_{k} \cdot \mathbf{b} & =0 \\
b_{j} & >0, \quad j=1, \ldots, m
\end{aligned}
$$


where $\mathbf{b}=\left[\begin{array}{lll}b_{1} & \ldots & b_{m}\end{array}\right]^{T}$.

For simplicity, we introduce the following notions. A Kirchhoff matrix is called weakly reversible if the corresponding reaction graph is weakly reversible. A vector $\mathbf{p} \in \mathbb{R}^{n}$ is called strictly positive if it is elementwise strictly positive, i.e. $p_{i}>0$ for $i=1, \ldots, n$. Two $n \times n$ matrices $A$ and $B$ are called structurally equal if the following is fulfilled: $A_{i j} \neq 0$ if and only if $B_{i j} \neq 0$ for $i, j=1, \ldots, n$. (Therefore, two structurally equal Kirchhoff matrices encode the same unweighted reaction graph structure.)

It can be seen that eq. (11) itself is a nonlinear constraint if both $A_{k}$ and $\mathbf{b}$ are unknowns. In order to formulate it as set of linear constraints, we can introduce a scaled Kirchhoff matrix $\tilde{A}_{k}$ as follows:

$$
\left[\tilde{A}_{k}\right]_{i, j}=\left[A_{k}\right]_{i, j} \cdot b_{j}, i, j,=1, \ldots, m
$$

Now, $A_{k}$ corresponds to a weakly reversible CRN if and only if $\mathbf{1}^{(m)}=\left[\begin{array}{llll}1 & 1 & \ldots & 1\end{array}\right]^{T} \in \mathbb{R}^{m}$ is an element of $\operatorname{ker}\left(\tilde{A}_{k}\right)$. Moreover, it is trivial that $\tilde{A}_{k}$ is weakly reversible if and only if the original Kirchhoff matrix $A_{k}$ is also a weakly reversible one. Based on these facts, the linear constraint set for weak reversibility can be formulated as follows:

$$
\begin{aligned}
& \sum_{i=1}^{m}\left[\tilde{A}_{k}\right]_{i, j}=0, \quad j=1, \ldots, m \\
& \sum_{i=1}^{m}\left[\tilde{A}_{k}\right]_{j, i}=0, \quad j=1, \ldots, m \\
& {\left[\tilde{A}_{k}\right]_{i, j} \geq 0, \quad i, j=1, \ldots, m, i \neq j,}
\end{aligned}
$$

where $A_{k}$ and $\tilde{A}_{k}$ are structurally equal.

\section{Known methods for computing weakly reversible CRN struc- tures with optimization}

In this Section we will shortly review two different existing MILP-based methods to find dynamically equivalent weakly reversible realizations of a CRN.

\subsection{One-step MILP procedure to compute weakly reversible realizations with additional preferred structural properties}

First, we briefly recall the part ensuring the structural equality of $A_{k}$ and $\tilde{A}_{k}$ of the algorithm presented in [16]. In that paper, a set of boolean decision variables are introduced and used as follows:

$$
\left[A_{k}\right]_{i, j}>\epsilon \leftrightarrow[\tilde{A}]_{i, j}>\epsilon, \quad i, j=1, \ldots, m, \quad i \neq j,
$$

where $\leftrightarrow$ means the 'if and only if' relation from classical binary logic and $\epsilon$ is a small fixed positive threshold value to distinguish between practically zero and nonzero edge weights in 
the reaction network. This logical condition can be expressed in the form of equivalent linear constraints (for the general framework, see e.g. [23]):

$$
\begin{aligned}
& 0 \leq\left[A_{k}\right]_{i, j}-\epsilon \delta_{i, j}, \quad i, j=1, \ldots, m, \quad i \neq j, \\
& 0 \leq-\left[A_{k}\right]_{i, j}+U B \cdot \delta_{i, j}, \quad i, j=1, \ldots, m, \quad i \neq j . \\
& 0 \leq\left[\tilde{A}_{k}\right]_{i, j}-\epsilon \delta_{i, j}, \quad i, j=1, \ldots, m, \quad i \neq j \\
& 0 \leq-\left[\tilde{A}_{k}\right]_{i, j}+U B \cdot \delta_{i, j}, \quad i, j=1, \ldots, m, \quad i \neq j,
\end{aligned}
$$

where $\delta_{i, j}, i, j=1, \ldots, m, i \neq j$ are boolean decision variables and $U B$ is the upper bound for the elements of $A_{k}$ and $\tilde{A}_{k}$. Due to the introduction of the $\delta$ variables, the resulting problem could be solved in the framework of MILP, making the handling of larger networks difficult. On the other hand, these boolean variables can be used to keep track of the presence of individual reactions, and by minimizing or maximizing the sum $\sum_{i, j=1}^{m} \delta_{i, j}$, a sparse or dense realization (containing the maximal or minimal number of reactions, respectively) can be obtained [6].

\subsection{Graph-theory inspired, iterative procedure to find dense weakly reversible realizations}

In [9] a completely different, graph-theory motivated method is presented (only for the case of dynamical equivalence) which leads to an iterative MILP-based algorithm. The algorithm requires $Y$ and $M$ as inputs, and computes an initial dense realization for them. Afterwards, it determines all the edges in the network that connect different strong components. In the next step, by solving an MILP problem a valid dense realization is determined without these edges (if it exists). These steps are repeated until the reaction graph of the resulting CRN is found to be weakly reversible. The algorithm ends with failure if there does not exist any dynamically equivalent realization that does not contain the directed edges to be excluded. In the original publication, an MILP problem is solved in each iteration step. Fortunately, as it is described in [10], the MILP problem for the computation of dense reaction structures can be safely replaced with a purely LP-based algorithm. This enables us to significantly speed up the solution process of the original method published in [9]. Therefore, we implemented and used the LP-based modified version of this graph-theory inspired algorithm for the present paper to compare its performance to our new methods. This algorithm will be shortly called the graph-based method in the paper.

\section{New results on the computation of weakly reversible CRN structures}

In this Section, we first present a theoretical result, then two new algorithms are introduced to compute weakly reversible realizations of CRNs. The first method can be applied to compute 
dynamically equivalent CRNs, while the second one can be used in the linearly conjugate case as well.

\subsection{The dense weakly reversible realization forms a super-structure for a fixed complex set}

It was shown in [6] that the dense realization is a unique superstructure containing all mathematically possible reactions, i.e for a given complex set, it contains all possible other realization structures as sub-graphs of the dense one. In the following, we will prove that a dense and weakly reversible realization contains all possible weakly reversible realizations if the complex set is fixed.

Theorem 1. Consider a kinetic system $\Sigma: \dot{\mathbf{x}}=M \cdot \psi(\mathbf{x})$. Suppose that $\left(Y, A_{k}\right)$ is a weakly reversible dynamically equivalent realization of $\Sigma$ that contains the maximal number of nonzero elements in $A_{k}$. Then, for any weakly reversible Kirchhoff matrix $A_{k}^{\prime}$ for which $Y \cdot A_{k}=Y \cdot A_{k}^{\prime}$ the following holds: $\left[A_{k}^{\prime}\right]_{i, j}>0$ implies $\left[A_{k}\right]_{i, j}>0$ for any $i \neq j$.

Proof. (by contradiction) Consider a weakly reversible Kirchhoff matrix $A_{k}^{\prime}$ for which $Y \cdot A_{k}=$ $Y \cdot A_{k}^{\prime}$. Suppose that there exists $1 \leq i, j \leq m, i \neq j$ for which $\left[A_{k}^{\prime}\right]_{i, j}>0$, but $\left[A_{k}\right]_{i, j}=0$. Let us define the matrix $\tilde{A}_{k}$ as

$$
\tilde{A}_{k}=\frac{A_{k}+A_{k}^{\prime}}{2}
$$

Clearly, $Y \cdot A_{k}=Y \cdot \tilde{A}_{k}$, and $\left[\tilde{A}_{k}\right]_{i, j}>0$. It follows from the weak reversibility of $A_{k}$ that there exists a strictly positive vector $\mathbf{p} \in \mathbb{R}^{m}$ such that $A_{k} \cdot \mathbf{p}=0$. Similarly, there exists a strictly positive vector $\mathbf{p}^{\prime}$ in the kernel of $A_{k}^{\prime}$, too. Let us define the following scaled Kirchhoff matrices: $\bar{A}_{k}=A_{k} \cdot \operatorname{diag}(\mathbf{p}), \bar{A}_{k}^{\prime}=A_{k}^{\prime} \cdot \operatorname{diag}\left(\mathbf{p}^{\prime}\right)$. Then $\bar{A}_{k}$ and $\bar{A}_{k}^{\prime}$ are Kirchhoff, and they are structurally equal to $A_{k}$ and $A_{k}^{\prime}$, respectively. Moreover, $\bar{A}_{k} \cdot \mathbf{1}^{(m)}=\bar{A}_{k}^{\prime} \cdot \mathbf{1}^{(m)}=0$, where $\mathbf{1}^{(m)}$ denotes the $m$ dimensional column vector composed of ones. Let $\hat{A}_{k}=\bar{A}_{k}+\bar{A}_{k}^{\prime}$. Then $\hat{A}_{k}$ is a weakly reversible Kirchhoff matrix, since $\hat{A}_{k} \cdot \mathbf{1}^{(m)}=\left(\bar{A}_{k}+\bar{A}_{k}^{\prime}\right) \cdot \mathbf{1}^{(m)}=0$. It is also clear that $\hat{A}_{k}$ is structurally equal to $\tilde{A}_{k}$. This implies that $\tilde{A}_{k}$ is a weakly reversible Kirchhoff matrix containing more non-zero off-diagonal elements than $A_{k}$, which is a contradiction.

To briefly illustrate the above theorem, consider a kinetic system $\Sigma: \dot{\mathbf{x}}=M \cdot \psi(\mathbf{x})$ characterized by the following matrices:

$$
Y=\left[\begin{array}{llll}
1 & 2 & 1 & 1 \\
2 & 1 & 3 & 1
\end{array}\right], \quad M=\left[\begin{array}{rrrr}
0 & -2 & 0 & 2 \\
-3 & 2 & -2 & 0
\end{array}\right]
$$

This kinetic system was studied before in [18] and in [9]. A possible dense weakly reversible 
realization $\left(Y, A_{k}^{(1)}\right)$ of $\Sigma$ is given by the Kirchhoff matrix:

$$
A_{k}^{(1)}=\left[\begin{array}{rrrr}
-3.2 & 1.8 & 0.1 & 0 \\
0 & -2 & 0 & 2 \\
0.1 & 0.1 & -1.05 & 0 \\
3.1 & 0.1 & 0.95 & -2
\end{array}\right] \text {. }
$$

On the other hand, the following Kirchhoff matrix encodes a complex balanced and thus weakly reversible realization $\left(Y, A_{k}^{(2)}\right)$ of $\Sigma$ (see [9]):

$$
A_{k}^{(2)}=\left[\begin{array}{rrrr}
-3 & 1.5 & 0 & 0 \\
0 & -2 & 0 & 2 \\
0 & 0.25 & -1 & 0 \\
3 & 0.25 & 1 & -2
\end{array}\right] .
$$

Finally, a sparse weakly reversible realization containing only 5 reactions $\left(Y, A_{k}^{(3)}\right)$ is defined by:

$$
A_{k}^{(3)}=\left[\begin{array}{rrrr}
-3.2 & 2 & 2 & 0 \\
0 & -2 & 0 & 2 \\
0.1 & 0 & -2 & 0 \\
3.1 & 0 & 0 & -2
\end{array}\right] .
$$

It can be easily checked that all $\left(Y, A_{k}^{(1)}\right),\left(Y, A_{k}^{(2)}\right)$ and $\left(Y, A_{k}^{(3)}\right)$ are dynamically equivalent weakly reversible realizations of $\Sigma$. The reaction graph structures of the three realizations are depicted in Fig. 1. It is clearly visible from the figure that the unweighted reaction graphs of $\left(Y, A_{k}^{(2)}\right)$ and $\left(Y, A_{k}^{(3)}\right)$ are indeed proper subgraphs of the unweighted reaction graph of $\left(Y, A_{k}^{(1)}\right)$.

\subsection{LP-based method to compute weakly reversible, dynamically equivalent realization}

In this subsection, an LP-based method is introduced which is able to compute a weakly reversible, dynamically equivalent realization for a given CRN. In a standard LP problem, a linear function of the real-valued optimization variables is minimized (or maximized) with respect to linear equality and inequality constraints. It is known that LP problems can be solved in polynomial time. Well-known solution approaches e.g. the simplex algorithm or interior-point methods are available in current software tools.

Let us denote the $i$ th column of $A_{k}$ and $M$ with $\mathbf{z}_{i}$ and $\mathbf{m}_{i}$, respectively. Then (8) can be written as

$$
Y \mathbf{z}_{i}=\mathbf{m}_{i}, \quad i=1, \ldots, m
$$


It is well-known from linear algebra that all solutions for eq. (22) can be characterized as the sum of particular solutions and the linear combinations of the solutions of the homogeneous system, that can be written as

$$
\mathbf{z}_{i}=\mathbf{z}_{i}^{(p)}+\sum_{j=1}^{r} \kappa_{i, j} \mathbf{z}_{j}^{(h)}, \quad i=1, \ldots, m
$$

where $\kappa_{i, j} \in \mathbb{R}, r$ is the nullity (i.e. kernel dimension) of $Y, \mathbf{z}_{i}^{(p)}$ is a particular solution for (22), and $\mathbf{z}_{j}^{(h)}$ is the $j$ th kernel base vector of $Y$. Since $Y$ and $M$ are given, $\mathbf{z}_{i}^{(p)}$ and $\mathbf{z}_{j}^{(h)}$ are known for $i=1, \ldots, m$ and $j=1, \ldots, r$. Thus, the unknowns in the problem will be the coefficients $\kappa_{i, j}$.

Recall eq. (11), namely that a CRN is weakly reversible if and only if there is a strictly positive vector $\mathbf{b}=\left[\begin{array}{lll}b_{1} & \ldots & b_{m}\end{array}\right]^{T}$ in the kernel of the matrix $A_{k}$. Then the condition (11) is given by

$$
\sum_{i=1}^{m} \mathbf{z}_{i} b_{i}=0
$$

Substituting (23) into (24) gives

$$
\sum_{i=1}^{m} \mathbf{z}_{i}^{(p)} b_{i}+\sum_{i=1}^{m} \sum_{j=1}^{r} b_{i} \kappa_{i, j} \mathbf{z}_{j}^{(h)}=0
$$

Let us introduce the following new variables

$$
v_{i, j}=b_{i} \kappa_{i, j}, \quad i=1, \ldots, m, \quad j=1, \ldots, r
$$

Using this notation, (25) reads

$$
\sum_{i=1}^{m} \mathbf{z}_{i}^{(p)} b_{i}+\sum_{i=1}^{m} \sum_{j=1}^{r} v_{i, j} \mathbf{z}_{j}^{(h)}=0
$$

that is now linear in the variables $b_{i}$ and $v_{i, j}$.

We are in a lucky situation considering the sign constraints of the elements of the original matrix $A_{k}$, since $\mathbf{b}$ is elementwise strictly positive (i.e. multiplying with $\mathbf{b}$ does not alter the signs of the elements in $A_{k}$ ). Let us denote by $z_{i, j}^{(p)}$ and $z_{i, j}^{(h)}$ the $j$ th scalar elements of the vectors $\mathbf{z}_{i}^{(p)}$ and $\mathbf{z}_{i}^{(h)}$, respectively. Then we can set the following constraints:

$$
\begin{aligned}
& z_{i, k}^{(p)} b_{i}+\sum_{j=1}^{r} v_{i, j} z_{j, k}^{(h)} \geq 0, \quad i, k=1, \ldots, m, \quad i \neq k \\
& z_{i, i}^{(p)} b_{i}+\sum_{j=1}^{r} v_{i, j} z_{j, i}^{(h)} \leq 0, \quad i=1, \ldots, m .
\end{aligned}
$$

The above method will be shortly referred to as $W R$-LP1 in the rest of the paper. For convenience and easy implementation, the steps of the $W R-L P 1$ algorithm are summarized in Table 1, where the input data are $Y$ and $M$, and the output is the Kirchhoff matrix of the computed weakly reversible dynamically equivalent realization if such exists, or 0 if the problem is infeasible. 


\subsection{LP-based method to compute linearly conjugate weakly reversible real- izations}

In this Section, we are going to present a new algorithm which is related to the MILP-based method briefly summarized in Section 3.1. That method is now extended to be able to deal with linear conjugacy and is also modified to eliminate the boolean decision variables from the model to obtain an LP-based algorithm. Let us assume that the matrix $M$ defined in eq. (8) containing the monomial coefficients of a kinetic system is given. It is known from [15] that linear conjugacy between two CRN models can be expressed by the following constraints:

$$
\begin{aligned}
& M=T \cdot Y \cdot A_{k} \\
& \sum_{i=1}^{m}\left[A_{k}\right]_{i, j}=0, \quad j=1, \ldots, m \\
& {\left[A_{k}\right]_{i, j} \geq 0, \quad i, j=1, \ldots, m, \quad i \neq j,} \\
& d_{i}>0, \quad i=1, \ldots, n,
\end{aligned}
$$

where the unknowns are the parameters of the positive diagonal transformation $T=\operatorname{diag}(\mathbf{d})$, and the off-diagonal elements of the Kirchhoff matrix $A_{k}$. The actual Kirchhoff matrix $A_{k}^{\prime}$ of the CRN realization that is linearly conjugate to the original kinetic system (9) defined by $M$ and $Y$, can be computed from $A_{k}$ and $\mathbf{d}$ using the following scaling (see [15] for the details):

$$
A_{k}^{\prime}=A_{k} \cdot \operatorname{diag}(\psi(\mathbf{d})) .
$$

Additionally, we use the auxiliary variable $\tilde{A}_{k}$ defined in (13) and constraints (14) to ensure weak reversibility, where again, $A_{k}$ and $\tilde{A}_{k}$ are structurally equal.

Using the fact that the off-diagonal elements of Kirchhoff matrices are non-negative, we can enforce the structural equality of $A_{k}$ and $\tilde{A}_{k}$ in our improved method using linear constraints without integer variables. Similarly to the solution in [16] (the constraints of which were summarized in eqs. (15)-(16)), we consider an off-diagonal element of a Kirchhoff matrix practically nonzero if it is greater than an appropriately chosen small positive value $p_{l}$ such that $0<p_{l} \ll 1$. (This means that off-diagonal elements less than $p_{l}$ are truncated to zero in $A_{k}$ and $\tilde{A}_{k}$.) Moreover, the following upper bounds are assumed for the off-diagonal elements of $A_{k}$ and $\tilde{A}_{k}$ with $p_{u}=\frac{1}{p_{l}}$.

$$
\left[A_{k}\right]_{i, j}<p_{u} \text { and }\left[\tilde{A}_{k}\right]_{i, j}<p_{u} \text { for } i, j=1, \ldots, m, \quad i \neq j .
$$

Now we set the following constraints for ensuring the structural equality of $A_{k}$ and $A_{k}^{\prime}$.

$$
\begin{aligned}
& {\left[A_{k}\right]_{i, j}-p_{u}^{2} \cdot\left[\tilde{A}_{k}\right]_{i, j} } \leq 0, \quad i, j=1, \ldots, m, \quad i \neq j \\
&-\left[A_{k}\right]_{i, j}+p_{l}^{2} \cdot\left[\tilde{A}_{k}\right]_{i, j} \leq 0, \quad i, j=1, \ldots, m, \quad i \neq j
\end{aligned}
$$

Let us examine the correctness of the constraints (36)-(37). For this, we have to take into account the upper bounds in (35), too. 
1. If $\left[A_{k}\right]_{i, j}>p_{l}$ and $\left[\tilde{A}_{k}\right]_{i, j}>p_{l}$ then one can see that $\left[A_{k}\right]_{i, j}<p_{u}^{2} \cdot\left[\tilde{A}_{k}\right]_{i, j}$ so eq. (36) is fulfilled. Moreover, because $\left[A_{k}\right]_{i, j}>p_{l}^{2} \cdot\left[\tilde{A}_{k}\right]_{i, j}$, eq. (37) holds, too.

2. If $\left[A_{k}\right]_{i, j}=0$ and $\left[\tilde{A}_{k}\right]_{i, j}=0$ then eqs. (36) and (37) are trivially fulfilled.

3. If $\left[A_{k}\right]_{i, j}>p_{l}$ and $\left[\tilde{A}_{k}\right]_{i, j}=0$ then eq. (36) is violated.

4. Similarly, if $\left[A_{k}\right]_{i, j}=0$ and $\left[\tilde{A}_{k}\right]_{i, j}>p_{l}$ then eq. (37) is violated.

In summary, the linear constraint set containing only continuous variables to find weakly reversible linearly conjugate $\mathrm{CRN}$ realizations is the following: (30) stands for the linear conjugacy, (31)-(32) encode the Kirchhoff property of $A_{k}$, and (33) ensures the positivity of the linear conjugacy transformation $T$. Constraints (14) introduce a scaled auxiliary Kirchhoff matrix $\tilde{A}_{k}$ that is weakly reversible, and finally, (36)-(37) ensure the structural equality of $A_{k}$ and $\tilde{A}_{k}$. The input data of the method are $Y$ and $M$, and the decision variables are the matrix elements $\left[A_{k}\right]_{i, j},\left[\tilde{A}_{k}\right]_{i, j}$ for $i, j=1, \ldots, m, i \neq j$, and the scaling factors $d_{k}$ for $k=1 \ldots, n$. Clearly, the feasibility of the constraints can be checked within the framework of linear programming. In this case, the objective function can be utilized to prescribe certain additional properties of the solution (if it exists). For example, to obtain a sparse weakly reversible realization of the studied kinetic system, the L1-norm of the elements of $A_{k}$ can be minimized, provided that the number of complexes in the CRN is large enough $[24,10]$. Later on, we will refer to this method as the $W R-L P 2$ algorithm.

\section{Computation results}

The capabilities of the algorithms presented in the previous section is illustrated through three examples. In the first one a dynamically equivalent weakly reversible realization does not exist but interestingly, a linearly conjugate, weakly reversible one does exist, and both cases are handled correctly by the applied computational method. The second example highlight a case where the algorithms are able to show the non-existence of a dynamically equivalent weakly reversible realization of a given CRN, as it is previously expected. Finally, the algorithms are compared in terms of computational time. All the computations were performed on a $2.6 \mathrm{GHz} \mathrm{PC}$ in MATLAB environment, using the CRNreals [25] and YALMIP [26] toolboxes. The CLP solver [27] was used to solve the LP problems, while the GLPK solver [28] was used to compare the results with the previously published MILP-based method [15]. The threshold to discriminate between zero and nonzero rate coefficients was set to $10^{-3}$.

Example 1 In this example which originally appeared in [16], a reaction network is shown which doesn't have a dynamically equivalent weakly reversible realization, but it has a linearly 
conjugate weakly reversible one. The network is characterized by

$$
Y=\left[\begin{array}{llllllllll}
1 & 2 & 2 & 2 & 1 & 2 & 1 & 2 & 1 & 1 \\
2 & 2 & 1 & 0 & 0 & 0 & 0 & 0 & 1 & 0 \\
0 & 0 & 0 & 0 & 0 & 1 & 2 & 2 & 2 & 1
\end{array}\right]
$$

and the Kirchhoff matrix $A_{k}$ containing the following non-zero off-diagonal elements: $\left[A_{k}\right]_{2,1}=1$, $\left[A_{k}\right]_{4,2}=0.5,\left[A_{k}\right]_{5,4}=1.5,\left[A_{k}\right]_{7,4}=0.5,\left[A_{k}\right]_{1,7}=0.5,\left[A_{k}\right]_{4,7}=1$. One can see that this $\left(Y, A_{k}\right)$ realization is non-reversible.

Firstly, while applying the presented $W R-L P 2$ algorithm on the above described system, we have fixed the $T$ matrix as an identity. This means that instead of looking for linearly conjugate realizations during the search, only the dynamically equivalent realizations were considered. The algorithm found the constraint set infeasible as expected.

Then, by relaxing the fixed value of the matrix $T$ we enabled the search for linearly conjugate realizations, too. Now the algorithm succeeded, determining a linearly conjugate weakly reversible realization with the following non-zero off-diagonal elements in the matrix $A_{k}^{(2)}$ : $\left[A_{k}^{(2)}\right]_{2,1}=0.001,\left[A_{k}^{(2)}\right]_{4,2}=0.005,\left[A_{k}^{(2)}\right]_{7,4}=0.002,\left[A_{k}^{(2)}\right]_{1,7}=0.005,\left[A_{k}^{(2)}\right]_{4,7}=0.001$ and linear conjugacy matrix $T=\operatorname{diag}\left(\left[\frac{1}{0.001}, \frac{1}{0.001}, \frac{1}{0.004}\right]\right)$. For these values, the equation for linear conjugacy $Y \cdot A_{k}=T \cdot Y \cdot A_{k}^{(2)}$ holds (see eq. (30)).

Example 2 In [2], the classical 3-dimensional kinetic Lorenz system that was described and studied in a CRN framework. With a proper parameter set the system is able to show chaotic behaviour. Due to the complex dynamics, we expect that this system will have neither a dynamically equivalent nor a linearly conjugate weakly reversible realization. In the following, it is shown that our algorithm actually returns this result with the given complex set and parameters.

By applying proper coordinates-shifting and an appropriate time-scaling (see [2] for the computation details), this system can be transformed to a kinetic form. The emerging kinetic ODEs are

$$
\begin{aligned}
& \dot{x}_{1}=\sigma x_{1} x_{2}^{2} x_{3}-\sigma x_{1}^{2} x_{2} x_{3}+\sigma\left(w_{1}-w_{2}\right) x_{1} x_{2} x_{3} \\
& \dot{x}_{2}=\left(\rho+c_{3}\right) x_{1}^{2} x_{2} x_{3}+\left(w_{2}-w_{1} \rho-w_{1} w_{3}\right) x_{1} x_{2} x_{3}-x_{1} x_{2}^{2} x_{3}-x_{1}^{2} x_{2} x_{3}^{2}+w_{1} x_{1} x_{2} x_{3}^{2} \\
& \dot{x}_{3}=x_{1}^{2} x_{2}^{2} x_{3}-w_{2} x_{1}^{2} x_{2} x_{3}-w_{1} x_{1} x_{2}^{2} x_{3}+\left(w_{1} w_{2}+\beta w_{3}\right) x_{1} x_{2} x_{3}-\beta x_{1} x_{2} \bar{x}_{3}^{2}
\end{aligned}
$$

The system described by eq. (38) can show chaotic behaviour (with an attractor that is very similar to the attractor of the classical non-kinetic Lorenz system) if the following parameter set is used [2]: $\sigma=10, \rho=28, \beta=8 / 3$, and $W=\left[\begin{array}{lll}w_{1} & w_{2} & w_{3}\end{array}\right]=\left[\begin{array}{ll}24 & 25 \\ 26\end{array}\right]$.

The complex composition matrix of the system is given by:

$$
Y^{(l)}=\left[\begin{array}{lllllllllllll}
1 & 0 & 2 & 1 & 2 & 1 & 1 & 1 & 2 & 2 & 2 & 1 & 2 \\
1 & 1 & 1 & 2 & 2 & 0 & 1 & 2 & 1 & 0 & 1 & 2 & 2 \\
1 & 1 & 1 & 1 & 1 & 1 & 2 & 2 & 2 & 2 & 0 & 0 & 2
\end{array}\right]
$$


while the non-zero off-diagonal elements of the network's Kirchhoff matrix $A_{k}^{(l)}$ are the following:

$$
\begin{aligned}
& {\left[A_{k}^{(l)}\right]_{2,1}=679.3324,\left[A_{k}^{(l)}\right]_{6,1}=1940.3342,\left[A_{k}^{(l)}\right]_{13,1}=669.3342,\left[A_{k}^{(l)}\right]_{11,3}=59,\left[A_{k}^{(l)}\right]_{12,3}=10,} \\
& {\left[A_{k}^{(l)}\right]_{13,3}=44,\left[A_{k}^{(l)}\right]_{10,4}=0.5,\left[A_{k}^{(l)}\right]_{12,4}=34,\left[A_{k}^{(l)}\right]_{13,4}=9.5,\left[A_{k}^{(l)}\right]_{13,5}=1,\left[A_{k}^{(l)}\right]_{8,7}=22.6666,} \\
& {\left[A_{k}^{(l)}\right]_{12,7}=1.3334,\left[A_{k}^{(l)}\right]_{10,9}=1 .}
\end{aligned}
$$

Now the monomial coefficient matrix can be written as

$$
M=Y^{(l)} \cdot A_{k}^{(l)}=\left[\begin{array}{ccccccccccccc}
-10 & 0 & -10 & 10 & 0 & 0 & 1 & 0 & 0 & 0 & 0 & 0 & 0 \\
-1271 & 0 & 54 & -1 & 0 & 0 & 24 & 0 & -1 & 0 & 0 & 0 & 0 \\
669.3342 & 0 & -25 & -24 & 1 & 0 & -2.6667 & 0 & 0 & 0 & 0 & 0 & 0
\end{array}\right]
$$

The $W R-L P 2$ algorithm found the problem infeasible during the search for dynamically equivalent and linearly conjugate weakly reversible realization. This coincides with the results of [2], where several thousand dynamically equivalent sparse realizations were computed with an efficient method, but none of them was weakly reversible.

Example 3 In this example containing several randomly generated CRNs, the results of the performance comparison of the presented algorithms are summarized while dealing with large scale networks. As it was shown, all three algorithms, namely the graph-based method, the $W R$ $L P 1$ and the WR-LP2 methods (presented in Section 3.2, Section 4.2 and 4.3, respectively) are purely LP-based algorithms. To be able to compare the three methods, only dynamically equivalent realizations were searched for.

All the algorithms were tested on a set of randomly generated CRNs. All the networks were built up from 10 species but contained different number of complexes: scenarios with 9, 30, 56, 90 complexes were set up, respectively. The methodology of generating the random kinetic systems was the following. Firstly, a kinetic polynomial system of the form (9) was generated where the elements of $M$ were uniformly distributed random real numbers from the interval $[10,110]$. The exponents of the monomials of $\psi$ were chosen as uniformly distributed random integers from the interval $[0,5]$. This kinetic polynomial system was converted to a so-called canonical CRN representation $\left(Y, A_{k}\right)$ as it is described in [20]. Then the obtained random CRN was extended with additional directed edges (if needed) to ensure that the resulting reaction graph is weakly reversible. This step was solved as an unweighted graph augmentation task $[29,30]$. The Kirchhoff matrix of the augmented weakly reversible network is denoted by $A_{k}^{\prime}$. The inputs for the realization computation algorithms were the matrices $Y$ and $M^{\prime}=Y \cdot A_{k}^{\prime}$.

The evaluation of the effectiveness of the algorithms - i.e. how the solution time is changing as the size of the computational task is growing - can be found in Table 2. The columns of the table show the size of the matrix $A_{k}$ (i.e. the number of complexes in the network) which basically determines the number of variables and constraints (depending also on the individual method). For each problem size, 10 different random CRNs were tested. In some cases the 
solver was unable to solve the problem in the given time limit (300s), these were considered as unsuccessful solution attempts. For any method, no incorrect solutions were obtained. Only the successful solutions were taken into account during the calculation of the average solution times. One can find the number of successful solutions (out of the original 10 problems for each problem size) in the corresponding rows of Table 2. In the remaining rows, the averaged solution times and the sizes of the generated LPs can be found for each algorithm.

One can see that despite the fact that both the WR-LP1 and the WR-LP2 algorithms solve a single LP problem, there is a significant difference between the solution times. This is caused by the different problem structures generated by the two algorithms. Although the number of variables and constraints is higher in the case of the WR-LP2 algorithm, the method generates a clear and sparse structure both for the equality and inequality constraints as it can be seen in Fig. 2, while WR-LP1 builds a nearly full coefficient matrix to describe the equality constraints (see Fig. 3). This fact has a serious effect on the computational time of the solution of the corresponding LP problems causing WR-LP1 not to terminate the computation within the predefined time limit in the case of larger networks.

For further comparison, we mention that the MILP-based method described in [15] produced the following results. In the case of the random networks with 9 complexes, we obtained 4 successful computation attemps (i.e. a correct solution within 300s) out of 10 attempts with an average solution time of $0.28 \mathrm{~s}$. For networks containing 30 complexes, the method gave only 1 successful solution attempt out of 10 with a solution time of 3.2s. For CRNs containing more than 30 complexes, the method did not give any correct solution within the given time limit.

\section{Conclusion}

We have developed and analyzed linear programming based methods to compute dynamically equivalent weakly reversible realizations of kinetic systems. Similarly to a result published in [8], it was shown that the dense dynamically equivalent weakly reversible realization structure of a kinetic system contains all other possible dynamically equivalent weakly reversible structures as proper subgraphs if the complex set is fixed. Based on the analysis of the properties of kinetic systems, a previously published graph-theory-based method was re-implemented without integer variables. In addition, two new computation methods were also proposed, having polynomial time complexity, too.

The implemented methods were tested on examples taken from the literature, and then they were compared from the point of view of computational performance on reaction networks of increasing size. The numerical tests showed that the LP-based methods solve the problems correctly, while avoiding the complexity issue which emerges during the solution of the former MILP-based problems. It was also shown that the structure of the constraint set in the LP problems has serious impact on the solution time of the problem. It clearly turned out that 
one method, called WR-LP2 (the only method that is able to handle linear conjugacy as well), outperforms the other LP-based methods in terms of computational time.

As a direction of possible future work, the proposed new methods can be developed further to incorporate additional requirements into the search for alternative CRN structures. These requirements can be any structural or parametrical properties which can be formulated as linear constraints. An interesting direction would be the inclusion of certain conservation laws into the search criteria.

\section{Acknowledgements}

This research has been supported by the Hungarian National Research Fund through the grants NF104706 and K83440. The first and second authors were also supported by the projects TÁMOP-4.2.1./B-11/2/KMR-2011-002 and TÁMOP-4.2.2./B-10/1-2010-0014.

\section{References}

[1] P. Érdi and J. Tóth. Mathematical Models of Chemical Reactions. Theory and Applications of Deterministic and Stochastic Models. Manchester University Press, Princeton University Press, Manchester, Princeton, 1989.

[2] Z. A. Tuza, G. Szederkényi, K. M. Hangos, and J. R. Banga A. A. Alonso. Computing all sparse kinetic structures for a Lorenz system using optimization methods. International Journal of Bifurcation and Chaos, accepted:to appear, 2013.

[3] W. M. Haddad, VS. Chellaboina, and Q. Hui. Nonnegative and Compartmental Dynamical Systems. Princeton University Press, 2010.

[4] N. Samardzija, L. D. Greller, and E. Wassermann. Nonlinear chemical kinetic schemes derived from mechanical and electrical dynamical systems. Journal of Chemical Physics, 90 (4):2296-2304, 1989.

[5] F. Horn and R. Jackson. General mass action kinetics. Archive for Rational Mechanics and Analysis, 47:81-116, 1972.

[6] G. Szederkényi. Computing sparse and dense realizations of reaction kinetic systems. Journal of Mathematical Chemistry, 47:551-568, 2010.

[7] G. Szederkényi and K. M. Hangos. Finding complex balanced and detailed balanced realizations of chemical reaction networks. Journal of Mathematical Chemistry, 49:1163-1179, 2011. 
[8] G. Szederkényi, K. M. Hangos, and T. Péni. Maximal and minimal realizations of reaction kinetic systems: computation and properties. MATCH Commun. Math. Comput. Chem., 65:309-332, 2011.

[9] G. Szederkényi, K. M. Hangos, and Zs. Tuza. Finding weakly reversible realizations of chemical reaction networks using optimization. MATCH Commun. Math. Comput. Chem., $67: 193-212,2012$.

[10] J. Rudan, G. Szederkényi, and K. M. Hangos. Efficiently computing alternative structures of large biochemical reaction networks using linear programming. MATCH Commun. Math. Comput. Chem., 71:71-92, 2014.

[11] M. Feinberg. Chemical reaction network structure and the stability of complex isothermal reactors - II. Multiple steady states for networks of deficiency one. Chemical Engineering Science, 43:1-25, 1988.

[12] D. F. Anderson. Boundedness of trajectories for weakly reversible, single linkage class reaction systems. Journal of Mathematical Chemistry, 49:1-16, 2011. DOI: 10.1007/s10910011-9886-4.

[13] J. Deng, C. Jones, M. Feinberg, and A. Nachman. On the steady states of weakly reversible chemical reaction networks. http://arxiv.org/abs/1111.2386, November 2011.

[14] B. Boros. On the existence of the positive steady states of weakly reversible deficiency-one mass action systems. Mathematical Biosciences, 245:157-170, 2013.

[15] M. D. Johnston, D. Siegel, and G. Szederkényi. A linear programming approach to weak reversibility and linear conjugacy of chemical reaction networks. Journal of Mathematical Chemistry, 50:274-288, 2012.

[16] M. D. Johnston, D. Siegel, and G. Szederkényi. Dynamical equivalence and linear conjugacy of chemical reaction networks: new results and methods. MATCH Commun. Math. Comput. Chem., 68:443-468, 2012.

[17] M. D. Johnston, D. Siegel, and G. Szederkényi. Computing weakly reversible linearly conjugate chemical reaction networks with minimal deficiency. Mathematical Biosciences, 241:88-98, 2013.

[18] M. D. Johnston and D. Siegel. Linear conjugacy of chemical reaction networks. Journal of Mathematical Chemistry, 49:1263-1282, 2011.

[19] M. Feinberg. Lectures on chemical reaction networks. Notes of lectures given at the Mathematics Research Center, University of Wisconsin, 1979. 
[20] V. Hárs and J. Tóth. On the inverse problem of reaction kinetics. In M. Farkas and L. Hatvani, editors, Qualitative Theory of Differential Equations, volume 30 of Coll. Math. Soc. J. Bolyai, pages 363-379. North-Holland, Amsterdam, 1981.

[21] V. Chellaboina, S. P. Bhat, W. M. Haddad, and D. S. Bernstein. Modeling and analysis of mass-action kinetics - nonnegativity, realizability, reducibility, and semistability. IEEE Control Systems Magazine, 29:60-78, 2009.

[22] G. Farkas. Kinetic lumping schemes. Chemical Engineering Science, 54:3909-3915, 1999.

[23] R. Raman and I.E. Grossmann. Modelling and computational techniques for logic based integer programming. Computers and Chemical Engineering, 18:563-578, 1994.

[24] D. L. Donoho and J. Tanner. Sparse nonnegative solution of underdetermined linear equations by linear programming. Proc. of the National Academy of Sciences of the USA (PNAS), 102(27):9446-9451, 2005.

[25] G. Szederkényi, J. R. Banga, and A. A. Alonso. CRNreals: a toolbox for distinguishability and identifiability analysis of biochemical reaction networks. Bioinformatics, 28(11):15491550, June 2012.

[26] J. Löfberg. YALMIP : A toolbox for modeling and optimization in MATLAB. In Proceedings of the CACSD Conference, Taipei, Taiwan, 2004.

[27] CLP - Coin-or linear programming.

https://projects.coin-or.org/Clp.

[28] GLPK - GNU Linear Programming Toolkit.

https://www.glpk.org.

[29] K. P. Eswaran and R. E. Tarjan. Augmentation problems. SIAM J. Comput., 5:653-665, 1976.

[30] S. Raghavan. The Next Wave in Computing, Optimization, and Decision Technologies, chapter 'A Note on Eswaran and Tarjan's Algorithm for the Strong Connectivity Augmentation Problem', pages 19-26. Kluwer Academic Press, 2005. 


\section{Figures}

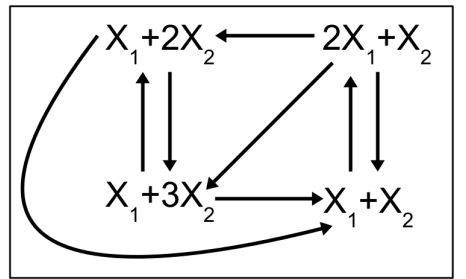

(a)

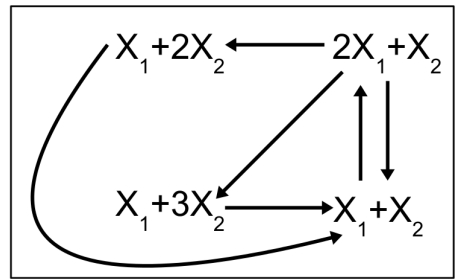

(b)

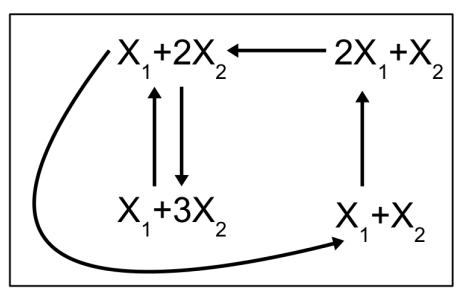

(c)

Figure 1: Possible dynamically equivalent weakly reversible reaction graph structures of the kinetic system (18). (a) Dense weakly reversible structure defined by $A_{k}^{(1)}$. (b) Weakly reversible structure of a complex balanced realization given by $A_{k}^{(2)}$. (c) Sparse weakly reversible structure with 5 reactions defined by $A_{k}^{(2)}$. 


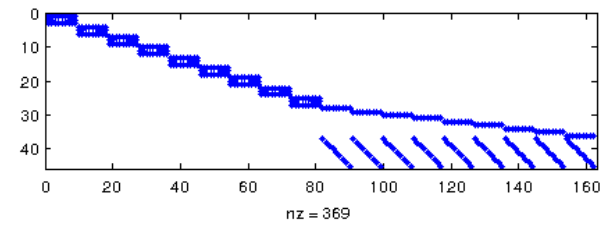

(a) Structure of the matrix describing the equality constraints in the LP problem. The size of the matrix is $45 \times 162$.

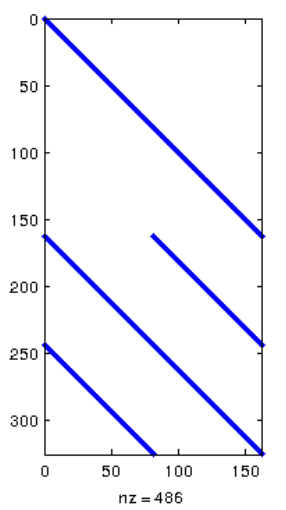

(b) Structure of the matrix describing the inequality constraints in the LP problem. The size of the matrix is $324 \times 162$.

Figure 2: Structure of the constraint set in case of the WR-LP2 algorithm. Rows and columns represent constraints and variables, respectively. The original CRN contains 2 species and 9 complexes. Non-zero elements of the matrices are marked. As one can note, the algorithm generates sparse constraint matrices with clear structure. 


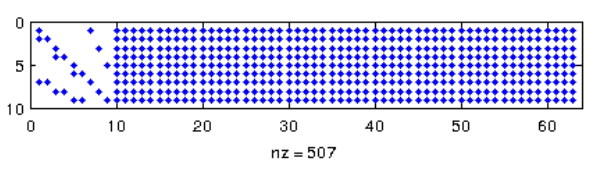

(a) Structure of the matrix describing the equality constraints in the LP problem. The size of the matrix is $9 \times 63$.

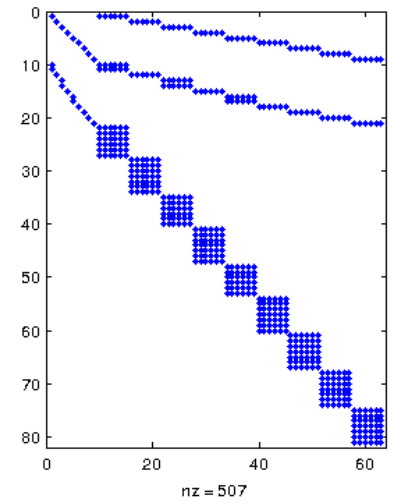

(b) Structure of the matrix describing the inequality constraints in the LP problem. The size of the matrix is $81 \times 63$.

Figure 3: Structure of the constraint set in case of the WR-LP1 algorithm. Rows and columns represent constraints and variables, respectively. The original CRN contains 2 species and 9 complexes. Non-zero elements of the matrices are marked. It can be seen that that the equality constraints formulate a nearly full matrix. 


\section{Tables}

$\frac{A_{k}=\mathrm{WR}-\mathrm{LP} 1(Y, M)}{1 \quad A_{k}:=0 \in \mathbb{R}^{m \times m}}$

2 Determine the particular and homogeneous solutions $z_{i}^{(p)}$

for $i=1, \ldots m$, and $z_{j}^{(h)}$ for $j=1, \ldots, r$ from eq. (22).

3 Check the feasibility of (25) and (28)-(29) with variables $\mathbf{b}$ and $v$ as a linear programming problem with arbitrary linear objective function.

4 If there exists a feasible solution:

$5 \quad$ Determine $\kappa_{i, j}$ from (26).

6 Compute the values of the original variable $A_{k}$ according to (23).

7 return $A_{k}$;

8 Else

9 return 0

Table 1: Steps of the method $W R-L P 1$ for finding weakly reversible, dynamically equivalent realization. 


\begin{tabular}{|c|c|c|c|c|}
\hline Network size $(m)$ & 9 & 30 & 56 & 90 \\
\hline \hline Graph-based & \multicolumn{4}{|l|}{} \\
\hline time (s) & 0.04 & 0.77 & 4.26 & 18.98 \\
\hline success & $10 / 10$ & $10 / 10$ & $10 / 10$ & $10 / 10$ \\
\hline \# of optim. vars ${ }^{*}$ & 14 & 37 & 65 & 101 \\
\hline \# of eq. constr. ${ }^{*}$ & 4 & 6 & 8 & 10 \\
\hline \# of ineq. constr. ${ }^{*}$ & 14 & 37 & 65 & 101 \\
\hline WR- $L P 1$ & \multicolumn{5}{|l|}{} \\
\hline time (s) & 0.001 & 2.04 & 5.98 & - \\
\hline success & $10 / 10$ & $10 / 10$ & $4 / 10$ & $0 / 10$ \\
\hline \# of optim. vars & 63 & 780 & 2800 & 7380 \\
\hline \# of eq. constr. & 9 & 30 & 56 & 90 \\
\hline \# of ineq. constr. & 81 & 900 & 3136 & 8100 \\
\hline WR- $L P 2$ & \multicolumn{5}{|l|}{} \\
\hline time (s) & 0.003 & 0.23 & 1.62 & 11.28 \\
\hline success & $10 / 10$ & $10 / 10$ & $10 / 10$ & $10 / 10$ \\
\hline \# of optim. vars & 162 & 1800 & 6272 & 16200 \\
\hline \# of eq. constr. & 45 & 210 & 504 & 990 \\
\hline \# of ineq. constr. & 324 & 3600 & 12544 & 32400 \\
\hline
\end{tabular}

Table 2: Comparison of the presented algorithms in terms of computational time while dealing with CRNs having different sizes. WR-LP2 algorithm outperforms all the other methods. All the compared methods are based on LPs as the constrained dense search is also implemented with LP for the graph-based method. The size of the generated LPs also appear in the table. *: the graph-based method calculates $m^{2}$ LPs with the given size. 